\title{
Simplification, aesthetics and performance in your hands
}

G-ænial A'CHORD is the latest addition to the G-ænial range, the culmination of ten years of clinical success, representing the ideal balance of simplicity, aesthetics and technology in one product. A significant advancement in composite materials, G-ænial A'CHORD is the product of two remarkable proprietary GC technologies: Full-Coverage Silane Coating (FSC) and High Performance Pulverised CERASMART (HPC). This superior technology enables unparalleled simplification and aesthetics: the G-ænial A'CHORD simplified unishade system has five core shades that achieves the aesthetics of the 16 classic Vita shades, delivering durable and invisible fillings with natural fluorescence.

G-ænial A'CHORD is designed for simplicity, giving dentists a versatile universal composite for anterior and posterior restorations. Its simplified unishade system means with five core shades you get the results of 16 classic Vita shades. Achieving first-class aesthetics with a reduced inventory and quick polishing makes it a time and cost-effective material for any modern practice. It also offers simplified handling, whether sculpting with a hand instrument or using a brush, due to its non-sticky Bis-MEPP monomer and optimised filler-monomer combination of proprietary FSC and HPC technology.
G-ænial A'CHORD excels in aesthetics with its natural fluorescence, created by its unique filler technology that mimics natural light reflection resulting in impressive invisible restorations. Now you can give your patients a truly confident smile that is beautifully invisible in any lighting conditions, including UV and near-UV light. Furthermore, it delivers a high gloss with minimal polishing, which saves time and wows patients. It retains this high gloss thanks to its specialised proprietary technology with uniform nano filler distribution and high filler load.

When presented with complex cases, G-ænial A'CHORD rises to the challenge with its extended portfolio of cervical, opaque, enamel and bleach shades. This makes it possible to create invisible fillings even for extreme challenges.

Another hallmark of G-ænial $A^{\prime} C H O R D$ is its unrivalled FSC and HPC technologies which gives the material its unique suite of characteristics and advantages. Firstly, the silane coating of the FSC creates a strong bond between particles in the resin matrix, resulting in a high wear resistance, colour retention and excellent radiopacity for long-lasting and natural-looking restorations.
Secondly, HPC filler technology enables improved handling with minimal gaps, providing substantial marginal integrity for virtually eliminating the occurrence of secondary caries. In addition, its ceramic content contributes to the material's durability and ease of handling.

Contact GC UK Ltd on 01908218999 , email info.uk@gc.dental or visit europe. gc.dental/en-GB/products/gaenialachord.

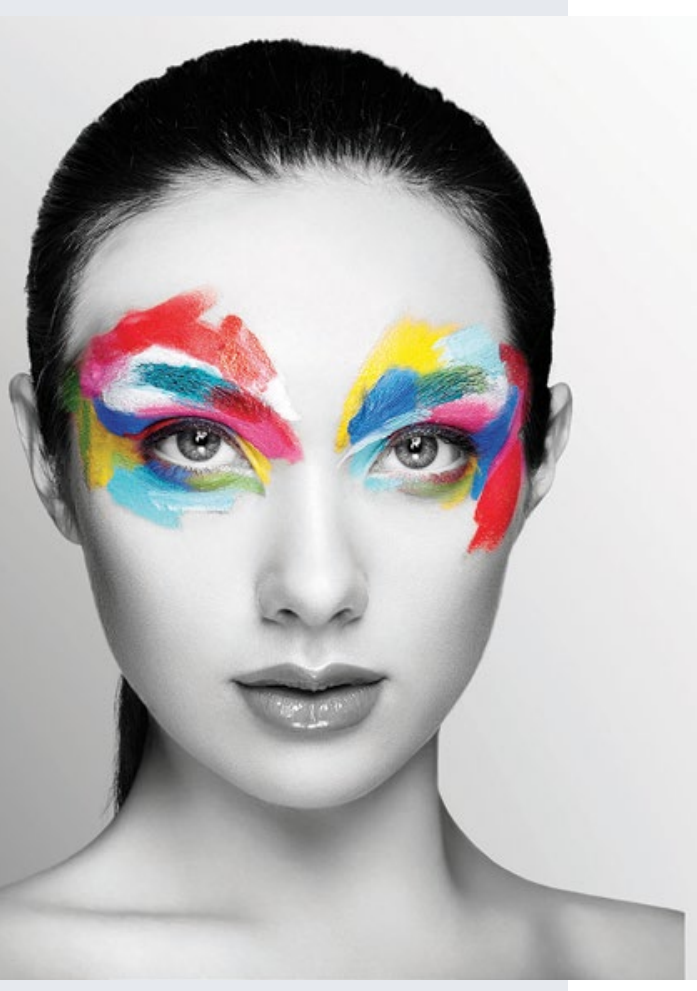

\section{Hygienic disposable products}

The American dental group Young Innovations has acquired British dental manufacturer Astek and is now taking over

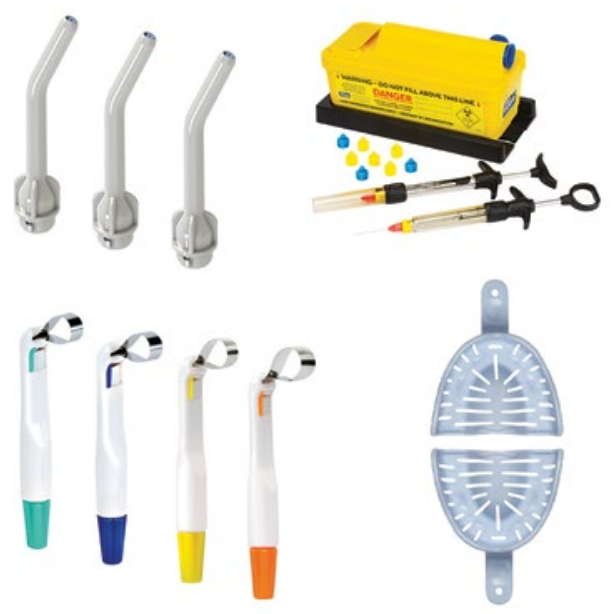

the distribution of the medical portfolio of Astek Innovations, including well-known brands Pro-Matrix, Pro-Tip, Transform, Pegasus, inSafe, and Alma Prosthetic Instruments.

Currently, the hygienic disposable products from Astek are in particular demand because they help to control infection risks. For example, the ready to use disposable matrix system ProMatrix, which does not require any tedious assembly and thus prevents injuries. The Pro-Matrix Curve range has a longer band for larger teeth and is precontoured to the tooth shape.

Effective infection control is also provided by the Pro-Tip Turbo disposable tips for air/water syringes. The clean separation of air and water in two chambers and single use significantly reduce the risk of cross-contamination. Another major health risk are needlestick injuries. The inSafe system ensures effective protection from the preparation of the syringe to the disposal of the used needle. More information in this video: https://www.youtube.com/ watch? $\mathrm{v}=\mathrm{d} 0 \mathrm{WksJv02 \textrm {o } 4 .}$

Another ground-breaking innovation is the thermoplastically mouldable impression tray Transform, made of biodegradable material. It was developed for prosthetic, orthodontic and implant procedures. It can be anatomically shaped and individually fitted within seconds.

https://www.astekinnovations.co.uk/ 